\title{
Relation between Helicobacter pylori infection and gastrointestinal symptoms and syndromes
}

\author{
S Rosenstock, L Kay, C Rosenstock, L P Andersen, O Bonnevie, T Jørgensen
}

Department of

Surgery K,

Bispebjerg University

Hospital,

Copenhagen,

Denmark

S Rosenstock

T Jørgensen

Glostrup University

Hospital,

Glostrup,

Denmark

S Rosenstock

L Kay

C Rosenstock

T Jørgensen

National University

Hospital,

Copenhagen,

Denmark

L P Andersen

Bispebjerg University

Hospital,

University of

Copenhagen,

Denmark

O Bonnevie

Correspondence to: Dr S Rosenstock, Department of Urology U, Frederiksberg Hospita

Nordre Fasanvej 57,

$2000 \mathrm{~F}$, Copenhagen,

Denmark.

Accepted for publication 20 February 1997

\begin{abstract}
Background-Helicobacter pylori is a human pathogen that colonises the gastric mucosa and causes permanent gastric inflammation.

Aims-To assess the symptoms of $\mathrm{H}$ pylori infection in an adult unselected population.

Subjects-A random sample of 3589 adult Danes who were examined in 1982 and 1987 ( $\mathrm{n}=2987$ ).

Methods-Abdominal symptoms within the preceding year were recorded at both attendances. Circulating IgG antibodies against $H$ pylori in serum samples drawn in 1982 were measured by using in-house indirect enzyme linked immunosorbent assays (ELISA).

Results-People with increased levels of IgG antibodies to $H$ pylori were more likely than uninfected individuals to report heartburn (odds ratio $(O R)=1.26$, 95\% confidence interval (CI) 1.03-1.54) and abdominal pain characterised by daily length $(\mathrm{OR}=1.33,95 \%$ CI $0.92-1.91)$, nocturnal occurrence $(O R=1.62,95 \% C I$ 1.19-2.19), spring aggravation $(O R=1.68$, 95\% CI $0.70-4.05)$, and no relation to meals $(\mathrm{OR}=0.62,95 \%$ CI $0.43-0.91)$ or stress $(O R=0.69,95 \%$ CI $0.50-0.95)$. The inclusion of people with increased levels of IgG antibodies to $H$ pylori, but without upper dyspepsia, at study entry significantly increased the likelihood of reporting upper dyspepsia at follow up (OR = $1.71,95 \%$ CI 1.24-2.36). People with epigastric pain and increased levels of IgM antibodies to $H$ pylori only indicative of acute $H$ pylori infection were more likely to report nocturnal pain, heartburn, nausea, and vomiting.
\end{abstract}

Conclusions - H pylori infection may precede the development of dyspepsia and is associated with a variety of gastrointestinal symptoms in people with no history of peptic ulcer disease.

(Gut 1997; 41: 169-176)

Keywords: epidemiology; Helicobacter pylori; non-ulcer dyspepsia; symptomatology; upper dyspepsia

Although the clinical picture of acute Helicobacter pylori infection has been described in detail in a few anecdotal reports, ${ }^{1-3}$ there is still considerable doubt as to whether chronic $H$ pylori infection causes gastrointestinal symptoms in people without peptic ulcer disease. ${ }^{45}$ Previous research regarding this issue has led to conflicting results. ${ }^{4}$ Some studies have shown that $H$ pylori infection rates are slightly higher in people with non-ulcer dyspepsia (NUD) than in asymptomatic people. ${ }^{6-10}$ Other groups have succeeded in linking specific gastrointestinal symptoms, such as postprandial bloating and belching, to the presence of $H$ pylori in the gastric mucosa. ${ }^{9-12}$ Finally, there have been reports of symptom relief in people with dyspepsia who were successfully treated for $H$ pylori infection, ${ }^{13}{ }^{14}$ but these results were questioned recently. ${ }^{15} 16$

Other studies have, however, failed to demonstrate any relation between $H$ pylori infection and gastrointestinal symptoms and specific $H$ pylori related dyspepsia has not yet been identified. ${ }^{8917-19}$ Moreover, attempts to relate $H$ pylori infection status to specific NUD subtypes, that is, reflux-like dyspepsia, ulcerlike dyspepsia, and dysmotility-like dyspepsia, have generally failed. ${ }^{14} 171920$

Assessment of the symptoms of acute $H$ pylori infection poses other problems. Acute infection occurs primarily in childhood and is rarely seen in adults. Most such cases remain undiagnosed. Consequently, it has been difficult to attain a consistent clinical image of this condition. Nevertheless, it is preferable that clinicians diagnose the infection immediately after the bacteria have colonised the gastric mucosa, as early diagnosis and treatment may lower the risk of peptic ulcer disease or gastric cancer later in life. ${ }^{21}$

The aim of this study was to assess the symptoms of serologically diagnosed acute and chronic $H$ pylori infections in an unselected adult population, with special reference to the prevalence and incidence of upper dyspepsia (UD).

\section{Materials and Methods}

STUDY POPULATION AND ACQUISITION OF SERUM SAMPLES

In October 1982 an age and sex stratified sample consisting of 4807 men and women, born in 1922, 1932, 1942, and 1952 (age 30, 40, 50, and 60 years), residing in the western part of Copenhagen County, was drawn from the National Danish Civil Registration System, in which all people living in Denmark are registered by a unique 10 -digit number. The distribution of sex, age, occupation, and marital status in the sampling area was compared with national statistics to ensure sample validity. ${ }^{22}$

All sample members received a standard letter containing information about the project and were invited to a general health examination. 
Also included was a questionnaire, to be completed in advance, ${ }^{22}$ concerning abdominal symptoms within the preceding year and previously diagnosed gastrointestinal disorders. Repeated requests were made in cases of nonresponse.

The sample size was reduced to 4581 Danes because 226 people of foreign extraction were excluded. Between November 1982 and February 19843608 people $(78.8 \%)$ entered the study. All responders had a general medical examination, including ultrasound assessment of the gall bladder. ${ }^{22}$ Serum samples were drawn from 3589 responders and stored at $-20^{\circ} \mathrm{C}$ pending analysis. There was a slight over-representation of tobacco smokers and people with short duration of schooling and poor social status in the non-responder group. ${ }^{23}$

After five years, all those attending the initial study were re-invited to a re-examination that was conducted between December 1987 and November 1988. One hundred and ten people had died and $85.4 \%$ of the eligible population $(n=2987 / 3498)$ attended the follow up examination. There was a significant difference in response rate among men because of a low attendance rate among 65 year olds, and a high attendance rate among those aged $45 .{ }^{24}$ Furthermore, non-responders were more likely than responders to be current tobacco smokers and originate from poor social strata. ${ }^{25}$ Medical examination, symptom questionnaires, and ultrasound assessment of the gall bladder were repeated. ${ }^{26}$

The project was approved by the Regional Research Ethics Commitee of Copenhagen County.

ANTIBODY DETECTION AND INTERPRETATION OF ANTIBODY PATTERNS

All serum samples drawn in 1982 were thawed for the first time and analysed in June 1993. Circulating IgG antibodies against a low molecular weight (LMW) fraction of $H$ pylori antigen ${ }^{27}$ and circulating $\operatorname{IgM}$ and $\operatorname{IgA}$ antibodies directed against heat stable (HS) $H$ pylori antigens were measured in duplicate by using an in-house indirect enzyme linked immunosorbent assay (ELISA). ${ }^{28}$ The IgG serology assay had previously been validated in 151 adult Danes with dyspeptic symptoms. This group was comparable to the present study population as regard sociodemographic factors and ethnicity. ${ }^{27}$ The sensitivity and specificity, at cut-off points $<100$ ELISA units (Eu) for seronegativity and $\geqslant 400$ Eu for seropositivity, were 98.5 and $54.0 \%$, respectively. The IgG serology assay predicted a history of peptic ulcer disease in this population when using the same cut-off points for seronegativity and seropositivity. ${ }^{29}$ Previous validation of the IgM serology assay in 167 persons with dyspepsia, of which 96 were $H$ pylori positive on biopsy based tests, showed a sensitivity of $67.7 \%$ and a specificity of $33.3 \%$ when cut-off points for seronegativity and seropositivity were assigned at $<100 \mathrm{Eu}$ and $\geqslant 200 \mathrm{Eu}$, respectively. ${ }^{28}$ Similar cut-off points for seronegativity and seropositivity were used for the IgG and the IgM serology in the present study.
For the IgA serology, cut-off points for seronegativity and seropositivity were assigned at $<100 \mathrm{Eu}$ and $\geqslant 100 \mathrm{Eu}^{29}$ Borderline cases were those with antibody levels between cut-off points for seronegativity and seropositivity. People with increased levels of IgG antibodies to $H$ pylori were assumed to harbour a chronic $H$ pylori infection regardless of $\operatorname{IgM}$ or $\operatorname{IgA}$ antibody values. Increased levels of IgM antibodies to $H$ pylori unaccompanied by an increase in IgG and IgA antibody levels were interpreted as a serological sign of acute $H$ pylori infection. ${ }^{1}$

ABDOMINAL SYMPTOMS WITHIN THE YEAR PRECEDING STUDY ENTRY IN 1982

All participants were asked about the occurrence of abdominal pain, heartburn, acid regurgitation, nausea, and vomiting within the year preceding study entry in 1982 (one year period prevalence). Except for abdominal pain, all abdominal symptoms were assessed on a four-point ordinal scale (never, occasionally, frequently, daily or almost constantly). Statements of occasional, frequent, or constant symptoms were considered affirmative answers and symptom scores were subsequently collapsed into simple dichotome variables (no, yes). Abdominal pain was recorded on a threepoint ordinal scale (never, occasionally, frequently). Subsequently, this scale was reduced to a simple dichotomous variable by categorising statements of occasional and frequent pain as affirmative answers. Abdominal pain was further characterised according to location (non-epigastric, epigastric), duration (minutes, hours, days, varying), seasonal occurrence (winter, spring, summer, autumn), nocturnal occurrence (no, yes), pain aggravating factors (no, eating, drinking, smoking, hunger, mental stress), and pain relieving factors (no, defaecation, eating, drinking, gastrointestinal drugs, non-opioid analgesics). Finally, a history of abdominal pain, that is, recurrent abdominal pain in the years preceding the year of study entry (life-time prevalence of abdominal pain), was treated as a dichotomous variable (no, yes).

\section{UPPER DYSPEPSIA DEFINITIONS}

Different definitions of upper dyspepsia (UD) were used in the present study. Firstly, the definition proposed by Colin-Jones was applied: upper abdominal pain, heartburn, nausea, vomiting, or other symptoms considered to be referrable to the proximal alimentary tract. ${ }^{30}$ Secondly, a UD classification based on the definition recently suggested by Talley ${ }^{31}$ (persistent or recurrent pain or abdominal discomfort centred in the upper abdomen) was tested. When using the latter definition, people with symptoms of gastro-oesophageal reflux disease (GORD) and people with concomitant irritable bowel syndrome (IBS) were not regarded as having UD. ${ }^{31}$ Finally, participants were diagnosed with UD if they had experienced at least one of the following symptoms: epigastric pain, heartburn, or acid regurgitation. ${ }^{32}$

UD prevalence refers to the percentage of participants who fulfilled these criteria at study 
entry in 1982, whereas incident cases of UD were those without UD at study entry who met the UD criteria at follow up in 1987.

REPRODUCIBILITY OF THE SYMPTOM

QUESTIONNAIRE

The reproducibility of the symptom questionnaire was tested in a sex and age stratified sample consisting of 170 participants drawn from the study population who were asked to complete an identical symptom questionnaire approximately two weeks after the first examination.

POSSIBLE CONFOUNDING FACTORS INCLUDED IN MULTIVARIATE ANALYSES

To avoid any possible confounding effects of other gastrointestinal disorders causing abdominal symptoms, analyses on the relation between abdominal symptoms, UD prevalence, and $H$ pylori infection were controlled for the effect of having biliary disease, IBS, or a history of peptic ulcer disease (PUD) at study entry. Likewise, analyses on the association between $H$ pylori infection and UD incidence were controlled for a history of incident cases of PUD, biliary disease, and IBS within the observation period. Only those who reported an ulcer verified by endoscopy, $x$-ray, or surgery were diagnosed as having PUD. ${ }^{33}$ By combining the outcome of the ultrasound assessment of the gall bladder with the participant's statements on prior cholecystectomy, at both attendances gall bladder status was classified as: normal, cholecystectomised, or gallstones. ${ }^{22}{ }^{26}$ Participants were diagnosed with IBS if they had abdominal pain and at least one of the following symptoms: changing stool consistency, borborygmi, or bloating. ${ }^{34}$

Information was obtained concerning sociodemographic factors, lifestyle practices, body mass index (BMI), and ingestion of nonsteroid anti-inflammatory drugs (NSAIDs) at study entry. ${ }^{35}$

STATISTICAL METHODS

The sPSs statistical package for Windows was used. ${ }^{36}$ The relation between $H$ pylori infection and upper gastrointestinal symptoms and syndromes was assessed in the entire study population. The symptomatology of acute $H$ pylori infection, as indicated by increased levels of IgM antibodies to $H$ pylori only, was examined in 333 participants who had epigastric pain at study entry.

Abdominal symptoms in 1982, UD prevalence in 1982, and UD incidence in 1987 were placed as the dependent variables in a series of logistic regression analyses. IgG and IgM antibody measurements were transformed into nominal scales according to cut-off levels (seronegative, borderline, seropositive) and used as explanatory variables. To obtain independent estimates of the impact of $H$ pylori infection on upper gastrointestinal symptoms, sex, age, BMI, current tobacco smoking, cumulated weekly alcohol consumption, daily coffee and tea intake, use of NSAIDs, familial social status, marital status, housing density, and occupational energy expenditure at study
TABLE 1 Seroprevalence $¥$ of increased IgG antibodies to $H$ pylori according to sex and age at study entry in 1982

\begin{tabular}{lll}
\hline \multirow{2}{*}{ Agef (years) } & \multicolumn{2}{l}{$I g G$ seropositivity \% $(n)^{*}$} \\
\cline { 2 - 3 } & Men & Women \\
\hline 30 & $12.9(451)$ & $13.7(454)$ \\
40 & $27.2(471)$ & $24.3(456)$ \\
50 & $25.6(461)$ & $30.4(448)$ \\
60 & $37.1(450)$ & $37.4(398)$ \\
All & $25.7(1833)$ & $26.1(1756)$ \\
\hline
\end{tabular}

${ }^{\star}$ Tests for trend with age: both significant with $\mathrm{p}$ values $<0.001$. †Age at the time of sampling in 1982.

$\ddagger$ Prevalence of increased IgG antibody levels $(\geqslant 400$ ELISA units) to $H$ pylori.

entry were subsequently included as possible confounding factors into multivariate logistic regression models, together with PUD, biliary disease, and IBS.

Odds ratios (ORs) were calculated as the natural antilogarithm of the coefficient $(\beta)$ for the separate variables of the fitted regression model. Ninety five per cent confidence intervals (CIs) for ORs were computed from the equation:

$$
C I=\left[e^{\beta \pm 1.96 \mathrm{SE}(\beta)}\right]
$$

where $\beta$ is the coefficient and SE is the standard error of the fitted model. The percentage of abdominal pain recordings that could be attributed to $H$ pylori infection (the population attributable risk per cent (PAR \%)) was calculated from the prevalence data by using the following equation:

$$
\mathrm{PAR} \%=\left(\mathrm{P}_{\mathrm{e}}(\mathrm{OR}-1) / \mathrm{P}_{\mathrm{e}}(\mathrm{OR}-1)+1\right) \times 100
$$

where $\mathrm{P}_{e}$ is the proportion of people with increased IgG antibody levels and OR is the odds ratio of abdominal pain in IgG seropositive people compared with non-infected individuals. ${ }^{37}$ The reproducibility of the symptom questionnaire was tested with the kappa statistic method. ${ }^{36}$

\section{Results}

REPRODUCIBILITY OF THE QUESTIONNAIRE All questionnaires from the 170 participants who received a second questionnaire were returned within an average time of 16.7 days (range nine to 43 days) after the first examination. The overall kappa value ( \pm 2 SD) was $0.75(0.71-0.79)$ and ranged from 0.57 (pain duration) to 0.89 (seasonal pain occurrence).

INCREASED LEVELS OF IgG ANTIBODIES TO $H$ PYLORI (CHRONIC H PYLORI INFECTION)

The prevalence of increased IgG antibody levels to $H$ pylori according to sex and age at study entry is shown in table 1 .

\section{Abdominal pain}

People with increased levels of IgG antibodies to $H$ pylori were more likely than non-infected individuals to have a history of abdominal pain and report abdominal pain within the preceding year (table 2). Sex had a significant interaction effect on these relations. Thus, having 
TABLE 2 One year and life-time prevalence of abdominal pain, abdominal pain characteristics and the seroprevalence of IgG antibodies to $H$ pylori in 1982: crude and multivariate adjusted odds ratios $(O R) \neq$

\begin{tabular}{|c|c|c|c|c|c|}
\hline $\begin{array}{l}\text { Symptoms and IgG antibody } \\
\text { status }\end{array}$ & $n(\%)$ & Unadjusted $O R$ & $\begin{array}{l}\text { Sex and age } \\
\text { adjusted } O R\end{array}$ & $\begin{array}{l}\text { Multivariate } \\
\text { adjusted OR }\end{array}$ & $95 \%$ CIS \\
\hline \multicolumn{6}{|c|}{ History of abdominal pain (life-time prevalence) } \\
\hline IgG seronegative & $248(12.5)$ & 1.0 & 1.0 & 1.0 & - \\
\hline IgG borderline & $84(12.4)$ & 0.99 & 0.99 & 0.92 & $(0.68-1.25)$ \\
\hline IgG seropositive & $165(17.8)$ & 1.51 & 1.47 & 1.18 & $(0.91-1.54)$ \\
\hline \multicolumn{6}{|c|}{ Abdominal pain within the preceding year (one-year period prevalence) ${ }^{\star}$} \\
\hline IgG seronegative & $229(11.5)$ & 1.0 & 1.0 & 1.0 & - \\
\hline IgG borderline & $74(10.9)$ & 0.94 & 0.96 & 0.89 & $(0.63-1.24)$ \\
\hline IgG seropositive & $128(13.8)$ & 1.23 & 1.27 & 1.23 & $(0.91-1.65)$ \\
\hline \multicolumn{6}{|l|}{ Epigastric pain } \\
\hline IgG seronegative & $356(42.1)$ & 1.0 & 1.0 & 1.0 & - \\
\hline IgG borderline & $123(40.9)$ & 0.95 & 0.89 & 0.89 & $(0.67-1.18)$ \\
\hline IgG seropositive & $188(45.2)$ & 1.13 & 0.99 & 0.92 & $(0.70-1.19)$ \\
\hline \multicolumn{6}{|l|}{ Nocturnal pain } \\
\hline IgG seronegative & $237(28.1)$ & 1.0 & 1.0 & 1.0 & - \\
\hline IgG borderline & $118(39.3)$ & 1.66 & 1.58 & 1.62 & $(1.19-2.19)$ \\
\hline IgG seropositive & $155(37.3)$ & 1.52 & 1.36 & 1.13 & $(0.85-1.51)$ \\
\hline \multicolumn{6}{|l|}{ Pain duration $\dagger$} \\
\hline Minutes & $153(32.0)$ & 1.0 & 1.0 & 1.0 & - \\
\hline Days & $101(43.7)$ & 1.65 & 1.53 & 1.33 & $(0.92-1.91)$ \\
\hline \multicolumn{6}{|l|}{ Factors aggravating pain } \\
\hline \multicolumn{6}{|l|}{ Spring ${ }^{\star}$} \\
\hline IgG seronegative & $45(51.7)$ & 1.0 & 1.0 & 1.0 & - \\
\hline IgG borderline & $19(51.4)$ & 0.99 & 1.05 & 1.20 & $(0.44-3.26)$ \\
\hline IgG seropositive & $44(67.7)$ & 1.96 & 2.14 & 1.68 & $(0.70-4.05)$ \\
\hline \multicolumn{6}{|l|}{ Autumn } \\
\hline IgG seronegative & $55(63.2)$ & 1.0 & 1.0 & 1.0 & _- \\
\hline IgG borderline & $29(78.4)$ & 2.11 & 2.01 & 3.29 & $(0.99-11.0)$ \\
\hline IgG seropositive & $42(64.6)$ & 1.06 & 0.97 & 0.68 & $(0.27-1.69)$ \\
\hline \multicolumn{6}{|l|}{ Drinking } \\
\hline IgG seronegative & $201(34.9)$ & 1.0 & 1.0 & 1.0 & - \\
\hline IgG borderline & $54(28.0)$ & 0.72 & 0.71 & 0.81 & $(0.55-1.20)$ \\
\hline IgG seropositive & $78(27.8)$ & 0.72 & 0.74 & 0.71 & $(0.50-1.01)$ \\
\hline \multicolumn{6}{|l|}{ Eating } \\
\hline IgG seronegative & $231(40.1)$ & 1.0 & 1.0 & 1.0 & - \\
\hline IgG borderline & $63(32.6)$ & 0.72 & 0.67 & 0.62 & $(0.43-0.91)$ \\
\hline IgG seropositive & $134(47.7)$ & 1.36 & 1.08 & 1.06 & $(0.76-1.47)$ \\
\hline \multicolumn{6}{|l|}{ Mental stress } \\
\hline IgG seronegative & $350(60.8)$ & 1.0 & 1.0 & 1.0 & - \\
\hline IgG borderline & $108(56.0)$ & 0.82 & 0.81 & 0.84 & $(0.59-1.20)$ \\
\hline IgG seropositive & $145(51.6)$ & 0.69 & 0.72 & 0.69 & $(0.50-0.95)$ \\
\hline
\end{tabular}

*Significant in women in multivariate analyses.

$\dagger \mathrm{IgG}$ antibody status was used as the dependent variable. Other pain episodes were not associated with $H$ pylori infection.

$\ddagger$ The upper odds ratio represents the risk of having the symptom in IgG borderline cases compared with IgG seronegative people. The lower odds ratio is the corresponding risk in IgG seropositive people compared with IgG seronegative people.

Confidence intervals for multivariate adjusted odds ratios.

ISignificant in men in multivariate analyses.

increased levels of IgG antibodies to $H$ pylori significantly increased the likelihood of reporting abdominal pain within the preceding year in women (multivariate adjusted OR 1.46 (1.01-2.14)), whereas this relation was not seen in men (multivariate adjusted OR 1.01 (0.63-1.61)). Seropositivity for IgG antibodies to $H$ pylori was associated with a history of abdominal pain in men (multivariate adjusted OR 1.59 (1.07-2.35)) but not in women (multivariate adjusted OR $0.93(0.65-1.34))$. The proportion of abdominal pain reports that could be attributed to $H$ pylori infection (the PAR \%) was $9.9 \%$ in men with a history of abdominal pain and $7.2 \%$ in women with abdominal pain within the preceding year.

\section{Abdominal pain characteristics}

When pain characteristics were assessed in participants who reported abdominal pain within the preceding year at study entry $(n=431)$, a pain pattern became evident in people with increased levels of IgG antibodies to $H$ pylori. IgG seropositive people most often described pain episodes that lasted for days and occurred predominantly at night. Pain was often aggravated in spring and was unlikely to be provoked by meals or mental stress (table 2). IgG antibody status was not associated with having pain that could be relieved by defaecation, eating, drinking, or medication.

Other symptoms referrable to the upper gastrointestinal tract

Whereas acid regurgitation and nausea were not associated with $H$ pylori infection, vomiting and heartburn were seen more often in IgG seropositive people than in non-infected individuals. The relation with heartburn persisted in multivariate analyses (IgG borderline versus IgG seronegative: OR 1.26 (1.03-1.54)).

\section{Prevalence and incidence of upper dyspepsia} Regardless of how UD was defined, the relation between UD prevalence and $H$ pylori infection did not reach significance (table 3 ). Nevertheless, there was a tendency towards higher UD incidence rates with all three UD definitions in people with increased levels of IgG antibodies and no sign of UD at study entry. When the authors' definition was examined, people with increased levels of IgG antibodies at study entry suffered a significant $71 \%$ increased risk of reporting UD at follow up when compared with IgG seronegative individuals despite control for first-time diagnosed ulcers within the same time period (table 3). 
TABLE 3 Prevalence of upper dyspepsia (UD) in 1982, incidence of UD in 1987 and seroprevalence of IgG antibodies to H pylori in 1982: crude and multivariate adjusted odds ratios (OR) $\neq$ by UD definitions *

\begin{tabular}{|c|c|c|c|c|c|}
\hline $\begin{array}{l}\text { UD definitions }{ }^{*} \text { and } \operatorname{Ig} G \\
\text { antibody status }\end{array}$ & $n(\%)$ & $\begin{array}{l}\text { Unadjusted } \\
\text { OR } \neq\end{array}$ & $\begin{array}{l}\text { Sex and age } \\
\text { adjusted OR } \neq\end{array}$ & $\begin{array}{l}\text { Multivariate } \\
\text { adjusted OR } \neq\end{array}$ & $95 \%$ CIt \\
\hline \multicolumn{6}{|l|}{ Colin-fones' definition } \\
\hline \multicolumn{6}{|l|}{ UD prevalence (1982) } \\
\hline IgG seronegative & $940(47.4)$ & 1.0 & 1.0 & 1.0 & - \\
\hline IgG borderline & $320(47.4)$ & 0.99 & 1.04 & 1.02 & $(0.84-1.24)$ \\
\hline IgG seropositive & $455(49.0)$ & 1.07 & 1.18 & 1.12 & $(0.93-1.35)$ \\
\hline \multicolumn{6}{|l|}{ UD incidence (1987) } \\
\hline IgG seronegative & $154(17.5)$ & 1.0 & 1.0 & 1.0 & - \\
\hline IgG borderline & $60(20.5)$ & 1.28 & 1.38 & 1.17 & $(0.78-1.75)$ \\
\hline IgG seropositive & $75(19.5)$ & 1.13 & 1.32 & 1.37 & $(0.96-1.97)$ \\
\hline \multicolumn{6}{|l|}{ Talley's definition } \\
\hline \multicolumn{6}{|l|}{ UD prevalence (1982) } \\
\hline IgG seronegative & $465(36.4)$ & 1.0 & 1.0 & 1.0 & - \\
\hline IgG borderline & $157(36.5)$ & 1.03 & 0.89 & 1.02 & $(0.80-1.30)$ \\
\hline IgG seropositive & $225(37.3)$ & 1.14 & 1.07 & 0.97 & $(0.78-1.21)$ \\
\hline \multicolumn{6}{|l|}{ UD incidence (1987) } \\
\hline IgG seronegative & $75(12.0)$ & 1.0 & 1.0 & 1.0 & - \\
\hline IgG borderline & $34(17.3)$ & 1.23 & 1.37 & 1.28 & $(0.86-1.91)$ \\
\hline IgG seropositive & $37(13.8)$ & 1.20 & 1.44 & 1.46 & $(1.02-2.10)$ \\
\hline \multicolumn{6}{|l|}{ Authors'definition } \\
\hline \multicolumn{6}{|l|}{ UD prevalence (1982) } \\
\hline IgG seronegative & $991(49.9)$ & 1.0 & 1.0 & 1.0 & - \\
\hline IgG borderline & $346(51.2)$ & 1.10 & 1.10 & 1.11 & $(0.92-1.34)$ \\
\hline IgG seropositive & $478(51.5)$ & 1.14 & 1.13 & 1.03 & $(0.86-1.23)$ \\
\hline \multicolumn{6}{|l|}{ UD incidence (1987) } \\
\hline IgG seronegative & $181(21.6)$ & 1.0 & 1.0 & 1.0 & - \\
\hline IgG borderline & $63(23.2)$ & 1.05 & 1.62 & 1.15 & $(0.80-1.65)$ \\
\hline IgG seropositive & $102(27.9)$ & 1.42 & 1.23 & 1.71 & $(1.24-2.36)$ \\
\hline
\end{tabular}

$\star$ See text for definition.

†95\% Confidence intervals for multivarite adjusted odds ratios.

$¥$ The upper odds ratio represents the risk of having the symptom in IgG borderline cases compared with IgG seronegative people. The lower odds ratio is the corresponding risk in IgG seropositive people compared with IgG seronegative people.

The corresponding figure was $46 \%$ when Talley's definition of UD was applied.

Prevalence and incidence of peptic ulcer disease, and $H$ pylori infection status at study entry People who were seropositive for IgG antibodies to $H$ pylori at study entry were more likely than uninfected individuals to have a history of PUD (prevalent ulcer) and to develop a first-time diagnosed (incident) ulcer within the five year observation period. When adjusted for possible confounders the odds ratios for prevalent and incident PUD were 4.2 (2.8-6.3) and 2.6 (1.1-6.2), respectively, for people with seropositive IgG antibody levels compared with people who were IgG seronegative.

INCREASED LEVELS OF IGM ANTIBODIES TO $H$ PYLORI ONLY (ACUTE H PYLORI INFECTION)

Gastrointestinal symptoms were not reported more often in people with increased levels of IgM antibodies to $H$ pylori only than in non-infected people. However, in a subgroup of 333 participants who had epigastric pain at study entry, nocturnal pain and heartburn were reported significantly more often in people with borderline increased levels solely of IgM antibodies to $H$ pylori than in non-infected individuals (table 4). Sex specific analyses, moreover, showed that men with borderline increased $\operatorname{IgM}$ antibodies to $H$ pylori were more likely than non-infected men to complain of nausea, vomiting, and pain that was induced by drinking. These relations were not seen in women (table 4).

\section{Discussion}

Numerous obstacles hamper the study of the relation between $H$ pylori infection and abdominal symptoms. Most cases of $H$ pylori infection are silent and, although abdominal symptoms are commonplace, not all dyspeptic patients seek medical advice. ${ }^{38}$ To examine the association between $H$ pylori infection and abdominal symptoms, unselected general populations rather than patients must be examined, as the latter may lead to Berkson's bias. ${ }^{39}$ Dyspeptic patients are liable to self-medicate with bismuth containing compounds, antacids, and antisecretory drugs that affect $H$ pylori infection rates. If $H$ pylori infection contributes little to the overall occurrence of abdominal symptoms, large-scale studies must be adopted. Non-invasive detection methods are necessary for large-scale screening for $\mathrm{H}$ pylori infection, ${ }^{40}$ but may not be as reliable as biopsy based methods. Symptom assessments must be meticulous as $H$ pylori infection may induce some abdominal symptoms while other symptoms may not be associated with the infection. Moreover, to assess the association between $H$ pylori infection and NUD, $H$ pylori related organic disease must be ruled out.

Whereas the sensitivity of the IgG serology used in this study was high, the specificity was relatively low. Consequently, some of the participants who tested IgG negative were probably infected with $H$ pylori. The low specificity would, however, mask a possible effect of $H$ pylori on gastrointestinal symptomatology as symptoms caused by $H$ pylori may also be reported by people who tested IgG seronegative. The diagnostic accuracy of commercially available serology kits varies considerably between different laboratories. ${ }^{41}$ The specificity of the present assay, however, was only slightly lower than that which could be obtained with commercially available detection kits in this study population. ${ }^{42}$ 
TABLE 4 Prevalence of abdominal symptoms in 333 Danes with epigastric pain at study entry in 1982 and seroprevalence of increased IgM antibodies to H pylori only: multivariate adjusted odds ratios (OR)*

\begin{tabular}{|c|c|c|c|c|c|c|}
\hline \multirow[b]{2}{*}{ Symptoms and IgM antibodies } & \multicolumn{3}{|c|}{$\operatorname{Men}(n=159)$} & \multicolumn{3}{|c|}{ Women $(n=174)$} \\
\hline & $n(\%)$ & $O R^{\star}$ & $95 \% \mathrm{CIt}$ & $n(\%)$ & $O R^{\star}$ & $95 \% \mathrm{CI}+$ \\
\hline \multicolumn{7}{|l|}{ Nocturnal pain $\neq$} \\
\hline IgG seronegative & $39(27.9)$ & 1.0 & & $49(32.5)$ & 1.0 & \\
\hline IgG borderline & $9(69.2)$ & 5.64 & $(1.12-28.39)$ & $7(43.8)$ & 1.88 & $(0.64-5.53)$ \\
\hline IgG seropositive & $0(0)$ & 0.00 & - & $3(42.9)$ & 1.53 & $(0.31-7.33)$ \\
\hline \multicolumn{7}{|l|}{ Pain aggravated by drinking } \\
\hline IgG seronegative & $50(45.5)$ & 1.0 & & $39(31.2)$ & 1.0 & \\
\hline IgG borderline & $9(75.0)$ & 3.98 & $(0.98-16.15)$ & $4(30.8)$ & 0.89 & $(0.16-4.80)$ \\
\hline IgG seropositive & $2(40.0)$ & 0.93 & $(0.14-6.06)$ & $3(50.0)$ & 2.16 & $(0.29-16.24)$ \\
\hline \multicolumn{7}{|l|}{ Nausea } \\
\hline IgG seronegative & $52(37.1)$ & 1.0 & & $81(53.6)$ & 1.0 & \\
\hline IgG borderline & $10(76.9)$ & 5.26 & $(1.30-21.33)$ & $9(56.3)$ & 0.77 & $(0.22-2.73)$ \\
\hline IgG seropositive & $0(0)$ & 0.00 & - & $2(28.6)$ & 0.60 & $(0.09-4.13)$ \\
\hline \multicolumn{7}{|l|}{ Vomiting } \\
\hline IgG seronegative & $24(17.1)$ & 1.0 & & $39(25.8)$ & 1.0 & \\
\hline IgG borderline & $7(53.8)$ & 4.73 & $(1.32-16.93)$ & $3(18.8)$ & 0.34 & $(0.07-1.79)$ \\
\hline IgG seropositive & $1(16.7)$ & 1.09 & $(0.10-11.55)$ & $0(0)$ & 0.00 & - \\
\hline \multicolumn{7}{|l|}{ Hearburn $\ddagger$} \\
\hline IgG seronegative & $75(53.6)$ & 1.0 & & $75(49.7)$ & 1.0 & \\
\hline IgG borderline & $10(76.9)$ & 3.95 & $(0.65-23.85)$ & $11(68.8)$ & 4.08 & $(0.90-18.43)$ \\
\hline IgG seropositive & $2(33.3)$ & 0.25 & $(0.03-2.43)$ & $3(42.9)$ & 0.81 & $(0.13-4.95)$ \\
\hline
\end{tabular}

«The upper odds ratio represents the risk of having the symptom in IgM borderline cases compared with IgM seronegative people. The lower odds ratio is the corresponding risk in IgM seropositive people compared with IgM seronegative people.

$+95 \%$ Confidence intervals for multivariate adjusted odds ratios.

$\ddagger$ Odds ratios were significant when men and women were grouped together (IgM borderline $><\operatorname{IgM}$ seronegative: OR nocturnal pain 2.43 (1.01-5.83), OR heartburn 4.11 (1.48-11.41)).

Talley recently pointed out that earlier studies in favour of a causal relation between $H$ pylori infection and abdominal symptoms may be biased by inappropriate study designs. ${ }^{15} 43$ The population based prospective cohort design applied in this study rules out a number of methodological flaws. The confounding effects of sociodemographic factors, lifestyle practices, and concomitant gastrointestinal disease were controlled for by multivariate logistic regression analyses. Valid assessment of abdominal symptoms is, however, essential as objective outcome measures do not exist. The reproducibility of the questionnaire was substantial as shown by the overall kappa statistic of 0.75 . The validity of the symptom questionnaire was previouly examined by comparing the one-year period prevalence of abdominal symptoms with symptom prevalences reported in other studies. Symptom prevalences were found to be valid for the study population. ${ }^{44}$ The symptom questionnaire was simple and written in comprehensible language. This method of obtaining data does not depart from the daily routine in clinical practice where doctors mostly accept the patients' answers to simple questions without further elaboration.

A high prevalence of PUD is found in $H$ pylori infected dyspeptic individuals. ${ }^{45}{ }^{46}$ The participants did not receive an endoscopic examination when they entered the study and, although gastroscopy is free of charge in Denmark, our results may be biased by the fact that some ulcers were missed. The prevalence of peptic ulcer disease in this study population ${ }^{33}$ was only slightly lower than ulcer rates found in a similar Norwegian population undergoing endoscopy because of dyspepsia. ${ }^{47}$ Therefore, the number of missed ulcers in this study is probably small.

$H$ pylori infection is found frequently in patients with dyspepsia undergoing endoscopy, but in whom no macroscopic lesions are found. Attention has, therefore, been directed to a possible role of $H$ pylori infections in the aetiology of NUD. In this study, $H$ pylori infection was associated with higher rates of heartburn, vomiting, and specific pain characteristics in people with no history of PUD, biliary disease, or IBS. This symptom cluster is not consistent with any of previously described NUD subtypes, but contains symptoms referrable to reflux-like dyspepsia (heartburn), and ulcerlike dyspepsia (nocturnal pain). ${ }^{30}$ The applicability of the NUD subtype definitions has been questioned previously. ${ }^{32} 48$ The present findings may further challenge the clinical relevance of this categorisation. Our results may also explain why attempts to link $H$ pylori infection to specific NUD subtypes have failed. ${ }^{14} 171920$

Seropositivity for IgG antibodies to $H$ pylori at study entry caused an appreciable increase in the risk of reporting UD at follow up in people who did not have UD when they entered the study. The temporal sequence points towards causality. This association reached significance with two of the UD definitions applied (table 2). No consensus has yet been reached on standard definitions of UD. ${ }^{30} 490$ The definition used by our group was previously applied to this population. UD prevalence was comparable to prevalence rates reported in studies from other Western countries. ${ }^{32}$ The increased risk of developing UD in people with $H$ pylori infection at study entry and the lack of significant association between $H$ pylori infection and UD prevalence is, however, of concern. H pylori infection is a progressive disease, which passes through a continuum of different histomorphological states. ${ }^{40}$ The duration of the infection could be the decisive factor influencing whether or not abdominal symptoms occur. ${ }^{21}$ The increase in symptom occurrence in $H$ pylori infected people was modest compared with people who had seronegative IgG antibody levels. Consequently, it could be inferred that the impact of eradicating an existing $H$ pylori infection in people with dyspepsia is lim- 
ited. Given that the relation between abdominal pain and $H$ pylori infection is causal, our results suggest that approximately $7-10 \%$ of all cases with abdominal pain would benefit from antimicrobial treatment.

As only a small number of $H$ pylori infected people develop dyspepsia, different factors must influence the clinical outcome of infection. Tytgat recently suggested that the extent and activity of the $H$ pylori associated gastritis may determine whether dyspepsia occurs. ${ }^{51}$ The intensity of gastritis probably reflects both host factors, such as genetic make-up and environmental setting, and bacterial factors, such as the virulence of the infecting strain. Strain specific differences in the relation between $H$ pylori infection and abdominal symptoms were not examined in this study. The lack of differentiation between different $H$ pylori strains may have resulted in random misclassifications, which inevitably weakens relations and may explain the small magnitude of the risk estimates.

The reports of the interaction of sex on abdominal pain are intriguing. Sex differences in intragastric acidity, se-gastrin, parietal cell mass, and gastric emptying have been reported in humans. ${ }^{52-54}$ The relation between $H$ pylori infection and these factors is not yet known in detail. Elaborations on the observed interactions, therefore, should await further research.

Symptoms associated with acute $H$ pylori infection are present only for a few weeks. ${ }^{1-3}$ As we assessed abdominal symptoms within a year, it is possible that symptoms caused by acute $H$ pylori infection were masked by other upper gastrointestinal symptoms unrelated to $H$ pylori infection. Abdominal symptoms were reported more often only in people with borderline increased IgM antibody levels but not in IgM seropositive people. The significance of borderline increased $\operatorname{IgM}$ antibody levels could, however, be questioned. Furthermore, the symptoms of acute $H$ pylori infection seen in men with epigastric pain shows a considerable overlap with symptoms of acute gastroenteritis. Cross-reacting antigens have been reported between $H$ pylori and Campylobacter jejuni, an organism known to cause gastroenteritis. ${ }^{55}$ A high cut-off level for IgM seropositivity was applied to lower the risk of including people with $C$ jejuni infections. Nevertheless, cross-reactions cannot be ruled out entirely.

No biological mechanism has yet been put forward that adequately explains how $H$ pylori may induce abdominal symptoms. Although gastric acid and gastrin secretion are increased in $H$ pylori infection, ${ }^{56}$ the relation between acid secretion and abdominal symptoms is undetermined..$^{57}$ The impact of $H$ pylori infection on gastric motility and gastric emptying likewise is equivocal. ${ }^{58}$ Pain mechanisms are not known in most gastrointestinal disorders, and this lack of biological mechanism may merely reflect current limitations of knowledge and is hardly an argument against a relation between $H$ pylori infection and abdominal symptoms.
In summary, $H$ pylori infection seems to precede the development of upper dyspepsia. Seropositivity for IgG antibodies to $H$ pylori relates to a variety of abdominal symptoms referrable to the upper gastrointestinal tract. The symptom cluster associated with IgG seropositivity in this population is not consistent with previously described NUD subtypes. $H$ pylori infection may give rise to different abdominal syndromes in men and women. Acute $H$ pylor $i$ infection in adults may be characterised by specific upper gastrointestinal symptoms, particularly in men.

This work was supported by grants from The Danish Medical Research Council (12-1844-1), The Clinical Foundation of the University of Copenhagen, The Ingeborg Roikjer Foundation (9043), The Danish Health Insurance Foundation (11/099-95), The Foundation of 1870 , the $\mathrm{Mr}$ and Mrs Ove Villiam Buhl Olesen Foundation (3555-11), and the $\mathrm{Mr}$ and Mrs Jacob Madsen Foundation. The technical assistance of Hanne Kubert and Susanne Rhode is greatly appreciated.

1 Sobala GM, Crabtree JE, Dixon MF, Schorah CJ, Taylor JD, Rathbone BJ, et al. Acute Helicobacter pylori infection: clinical features, local and systemic immune response, gastric mucosal histology, and gastric juice ascorbic acid concentrations. Gut 1991; 32: 1415-8.

2 Morris A, Nicholson G. Ingestion of Campylobacter pyloridis causes gastritis and raised fasting gastric $\mathrm{pH} . \mathrm{Am}$ 7 Gastroenterol 1987; 82: 192-9.

3 Marshall BJ, Armstrong JA, McGechie DB, Glancy RJ. Attempt to fulfil Koch's postulates for pyloric campylobacter. Med f Aust 1985; 142: 436-9.

4 Tytgat GNJ, Noach LA, Rauws EAJ. Is gastroduodenitis a cause of chronic dyspepsia? Scand $\mathcal{F}$ Gastroenterol 1991; 26 (suppl 182): 33-9.

5 Talley NJ. The Role of Helicobacter pylori in nonulcer dyspepsia. A debate-against. Gastroenterol Clin North Am 1993; 22: 153-67.

6 Shallcross TM, Rathbone BJ, Heatley RV. Campylobacter pylori and non-ulcer dyspepsia. In: Rathbone BJ, Heatley RV, eds. Campylobacter pylori and gastroduodenal disease. Oxford: Blackwell Science Publishers, 1989: 155-66.

7 Rauws EA, Langenberg W, Houthoff HJ, Zanen HC, Tytgat GNJ. Campylobacter pyloridis-associated chronic active antral gastritis. A prospective study of its prevalence and the effects of antibacterial and antiulcer treatment. Gastroenterology 1988; 94: 33-40.

8 Bernersen B, Johnsen R, Bostad L, Straume B, Sommer A-I, Burhol PG. Is Helicobacter pylori the cause of dyspepsia? BMF 1992; 304: 1276-9.

9 Strauss RM, Wang TC, Kelsey PB, Compton CC, Ferraro M-J, Perez-Perez G, et al. Association of Helicobacter pylori infection with dyspeptic symptoms in patients pylori infection with dyspeptic symptoms in patients
undergoing gastroduodenoscopy. Am $\mathcal{F}$ Med 1990; 89: 464-9.

10 Rokkas T, Pursey C, Uzoechina E, Dorrington L, Simmons NA, Filipe MI, et al. Campylobacter pylori and non-ulcer dyspepsia. Am f Gastroenterol 1987; 82: 1149-52.

11 Parsonnet J, Blaser MJ, Perez-Perez GI, Hargrett-Bean N, Tauxe RV. Symptoms and risk factors of Helicobacter pylori infection in a cohort of epidemiologists. Gastroenterology 1992; 102: 41-6.

12 Vaira D, Holton J, Osborn J, D'Anna L, Romanos A, Falzon M, et al. Endoscopy in dyspeptic patients: is gastric mucosal biopsy useful? Am f Gastroenterol 1990; 85: 701-4.
O'Morain CA, Buckley M. H. pylori in asymptomatic people. In: Hunt RH, Tytgat GNJ, eds. Helicobacter pylori. Basic mechanisms to clinical cure. Dordrecht/Boston/London: Kluwer Academic Publishers, 1994: 413-21.

14 Trespi E, Broglia F, Villani L, Luinetti O, Fiocca R, Solcia E. Distinct profiles of gastritis in dyspepsia subgroups. Their different clinical responses to gastritis healing after Helicodifferent clinical responses to gastritis healing after Helicob84-8.

15 Talley NJ. A critique of therapeutic trials in Helicobacter pylori-positive functional dyspepsia. Gastroenterology 1995; 106: $1174-83$

16 Veldhuyzen van Zanten SJO, Sherman PM. Helicobacter pylori infection as a cause of gastritis, duodenal ulcer, gastric cancer and nonulcer dyspepsia: a systematic overview. Can Med Assoc F 1994; 150: 177-85.

7 Holtmann G, Goebell H, Holtmann M, Talley NJ. Dyspepsia in healthy blood donors. Pattern of symptoms and association with Helicobacter pylori. Dig Dis Sci 1994; 39: 1090-8

18 Schubert TT, Schubert AB, Ma CK. Symptoms, gastritis, and Helicobacter pylori in patients referred for endoscopy. Gastrointest Endosc 1992; 38: 357-60.

19 Agréus L, Engstrand L, Svärdsudd K, Nyrén O, Tibblin G. Helicobacter pylori seropositivity among Swedish adults with and without abdominal symptoms. A populationwith and without abdominal symptoms. A populationb52-7.

20 Tucci A, Corinaldesi R, Stanghellini V, Tosetti C, Di Febo G, Paparo GF, et al. Helicobacter pylori infection and gas- 
tric function in patients with chronic idiopathic dyspepsia. Gastroenterology 1992; 103: 768-74.

21 Blaser MJ, Chyou P-H, Nomura A. Age at establishment of Helicobacter pylori infection and gastric carcinoma, gastric ulcer, and duodenal ulcer risk. Cancer Res 1995; 55: 562-5.

22 Jørgensen T. Prevalence of gallstones in a Danish population Am T. Prevalence of gallstones in a

23 Kirchhoff M. Hjerte-karsygdommenes udbredelse. Sociale og psykosociale forskelle i risikofaktorernes fordeling [Thesis]. Copenhagen: Københavns Universitet, 1988.

24 Rosenstock SJ. The Epidemiology of peptic ulcer disease in a Danish county [Thesis]. Copenhagen, Arhus, Odense: FADL Publishers, 1996: 1-155.

25 Kirchhoff $M$, Hansen B. Changes in smoking habits in a Danish population from 1983 to 1988 [abstract in English]. Ugeskrift für Lager 1995; 157: 3456-61.

26 Hougaard Jensen K, Jørqensen T. Incidence of gallstones in a Danish population. Gastroenterology 1991; 100: 790-4.

27 Andersen LP, Espersen F, Souckova A, Sedlackova M Soucek A. Isolation and preliminary evaluation of a low molecular (LMW) antigen preparation for improved detecmolecular (LMW) antigen preparation for improved detection of Helicobacter pylori IgG

28 Andersen LP, Raskov H-H, Elsborg L, Holck S, Justesen T, Hansen BF, et al. Prevalence of antibodies against heat-stable antigens from Helicobacter pylori in patients with dyspeptic symptoms and normal persons. APMIS 1992; 100: 779-89.

29 Andersen LP, Rosenstock SJ, Bonnevie O, Jørgensen T Seroprevalence of immunoglobulin G, M, and A antibodies to Helicobacter pylori in an unselected Danish population. Am f Epidemiol 1996; 143: 1157-64.

30 Colin-Jones DG. Management of dyspepsia: report of working party. Lancet 1988 ; i: 576-9.

31 Talley NJ, Colin-Jones DG, Koch KL, Koch M, Stanghellini V, Nyrén O. Functional dyspepsia: a classifica guidelines for diagnosis and management. Gastroenterology International 1991; 4: 145-60.

32 Kay L, Jørgensen T. Epidemiology of upper dyspepsia in a random population. Scand 7 Gastroenterol 1994; 29: 1-6.

33 Rosenstock SJ, Jørgensen T. Prevalence and incidence of peptic ulcer disease in a Danish county-a prospective cohort study. Gut 1995; 36: 819-24.

34 Kay L, Jørgensen T, Hougaard Jensen K. The epidemiology of irritable bowel syndrome in a random population: prevalence, incidence, natural history and risk factors. F Intern Med 1994; 236: 23-30.

35 Rosenstock SJ, Andersen LP, Rosenstock C, Bonnevie O, Jørgensen T. Socio-economic factors in Helicobacter pylor infection among Danish adults. Am f Public Health 1996; 86: $1539-44$.

36 Norusis MJ. SPSS for windows. Advanced statistics release 6.0, 1st edn. Chicago: SPSS Inc., 1993: 2-30.

37 Hennekens $\mathrm{CH}$, Buring JE. Epidemiology in medicine, 1st edn. Boston/Toronto: Little, Brown and Company, 1987.

38 Jones R, Lydeard S. Prevalence of symptoms of dyspepsia in the community. BMF 1989; 298: 30-2.

39 Roberts R, Spitzer W, Delmore T, Sackett D. An empirical demonstration of Berkson's bias. Fournal of Chronic Diseases 1978; 32: 119-28.

40 Taylor DN, Blaser MJ. The epidemiology of Helicobacter pylori infection. Epidemiol Rev 1991; 13: 42-59.

41 Feldman RA, Deeks JJ, Evans SJW, the Helicobacter pylor Serology Study Group. Multi-laboratory comparison of eight commercially available Helicobacter pylori serology kits. Eur F Clin Microbiol Infect Dis 1995; 14: 428-33.
42 Jensen AKV, Andersen LP, Wachmann $\mathrm{CH}$. Evaluation of eight commercial kits for Helicobacter pylori IgG antibody eight commercial kits for Helicobacter pyld

43 Talley NJ, Noack KB, The worldwide prevalence of Helicobacter pylori: asymptomatic infection and clinical states associated with infections in adults. In: Stewart Goodwin C, Worsley BW, eds. Helicobacter pylori biology and clinical practice. Boca Raton, Ann Arbor, London, Tokyo: CRC Press, 1993: 63-83.

44 Kay L, Jørgensen T, Hougaard Jensen K. Epidemiology of abdominal symptoms in a random population. Prevalence, incidence, and natural history. Eur f Epidemiol 1994; 10: 559-66.

45 Patel P, Khulusi S, Mendall MA, Lloyd R, Jazrawi RP, Maxwell JD, et al. Prospective screening of dyspeptic patients by Helicobacter pylori serology. Lancet 1995; 346: 1315-8.

46 Mendall MA, Jazrawi RP, Marrero JM, Molineaux N, Levi F, Maxwell JD, et al. Serology for Helicobacter pylori compared with symptom questionnaires in screening before direct access endoscopy. Gut 1995; 36: 330-3.

47 Johnsen R, Bernersen B, Straume B, Førde O, Bostad L, Burhol P. Prevalences of endoscopic and histological findings in subjects with and without dyspepsia. BMF 1991; 302: 749-52.

48 Agréus L, Svärdsudd K, Nyrén O, Tibblin G. Irritable bowel syndrome and dyspepsia in the general population: overlap and lack of stability over time. Gastroenterology 1995; 109: 671-80.

49 Kay L, Jørgensen T. Redefining abdominal syndromes. Results of a population-based study. Scand $\mathcal{F}$ Gastroenterol 1997 ; in press.

50 Drossman DA, Thompson WG, Talley NJ, Funch-Jensen P, Jansens J. Identification of subgroups of functional gastrointestinal disorders. Gastroenterology International 1990; 3: 159-72.

51 Tytgat GNJ. The clinical significance of Helicobacter pylori infection in chronic dyspepsia and peptic ulcer disease. Ann Med 1991; 23: 355-6.

52 Adeniyi KO. Gastric acid secretion and parietal cell mass: effect of sex hormones. Gastroenterology 1991; 101: 66-9.

53 Hutson WR, Roehrkasse RL, Wald A. Influence of gender and menopause on gastric emptying and motility. Gastroenterology 1989; 96: 11-7.

54 Prewett EJ, Smith JTL, Nwokolo CU, Sawyerr AM, Pounder RE. Twenty-four hour intragastric acidity and plasma gastrin concentration profiles in female and male subjects. Clin Sci 1991; 80: 619-24.

55 Newell DG. Identification of the outer membrane proteins of Campylobacter pyloridis and antigenic cross reactivity between C. pyloridis and C. jejuni. F Gen Microbiol 1987; 133: 163-70.

$56 \mathrm{McColl} \mathrm{KEL,} \mathrm{El-Omar} \mathrm{E.} \mathrm{Effect} \mathrm{of} \mathrm{H.} \mathrm{pylori} \mathrm{infection} \mathrm{on}$ gastrin and gastric acid secretion. In: Hunt RH, Tytgat GNJ, eds. Heliobacter pylori. Basic mechanisms to clinical cure. Dordrecht, Boston, London: Kluwer Academic Publishers, 1994: 245-56.

57 McColl KEL, Fullarton GM. Duodenal ulcer pain-the role of acid and inflammation. Gut 1993; 34: 1300-2

58 Caballero-Plasencia AM, Muros-Navarro MC, Martín-Ruiz JL, Valenzuela-Barranco M, De Los Reyes-García MC, Casado-Caballero FJ, et al. Dyspeptic symptoms and gastric emptying of solids in patients with functional dyspepsia. Role of Helicobacter pylori infection. Scand $\mathcal{F}$ Gastroenterol 1995; 30: 745-51. 